Published in final edited form as:

ACS Chem Neurosci. 2019 February 20; 10(2): 991-995. doi:10.1021/acschemneuro.8b00280.

\title{
Noncovalent, Electrostatic Interactions Induce Positively Cooperative Binding of Small Molecules to Alzheimer's and Parkinson's Disease-Related Amyloids
}

\author{
Jessica L. Cifelli, Christina C. Capule, and Jerry Yang ${ }^{*}$ \\ Department of Chemistry and Biochemistry, University of California, San Diego, 9500 Gilman \\ Drive, La Jolla, California 92093-0358, United States
}

\begin{abstract}
Amyloids are self-assembled protein aggregates that represent a major hallmark of many neurologic and systemic diseases. Among the common features of amyloids is the presence of a high density of multiple binding sites for small molecule ligands, making them an attractive target for design of multimeric binding agents. Here, we demonstrate that noncovalent, intermolecular interactions between a 1:1 mixture of oppositely charged benzothiazole molecules enhances their binding to two different amyloid aggregates: Alzheimer's-related amyloid- $\beta(\mathrm{A} \beta)$ peptides or Parkinson's-related $a$-synuclein ( $a \mathrm{~S}$ ) proteins. We show that this mixture leads to positively cooperative binding to amyloid targets, with up to 10-fold enhancement of binding compared to the uncharged parent compound. The observed enhancement of amyloid binding using noncovalent interactions was similar in magnitude to a benzothiazole dimer to aggregated $\mathrm{A} \beta$. These results represent a novel strategy for designing amyloid-targeting molecules with enhanced affinity, which could aid in the development of new diagnostic or treatment strategies for amyloidassociated diseases.
\end{abstract}

\section{Graphical Abstract}

\footnotetext{
*Corresponding Author Phone: 858-534-6006. Fax: 858-534-4554. jerryyang@ucsd.edu. Author Contributions

J.L.C. and J.Y. designed the research and analyzed the data. J.L.C. and C.C.C. executed the experiments. J.L.C. and J.Y. wrote the manuscript.

ASSOCIATED CONTENT

Supporting Information

The Supporting Information is available free of charge on the ACS Publications website at DOI: 10.1021/acschemneur-o.8b00280. Additional details for the synthesis and characterization of negatively charged (-)BAM1-EG6 and positively charged (+)BAM1-EG6, for preparation and characterization of amyloid aggregates, and for methods used to quantify additional binding characteristics; additional raw binding data (PDF)

The content is solely the responsibility of the authors and does not necessarily represent the official views of the National Institutes of Health.

The authors declare no competing financial interest.
} 


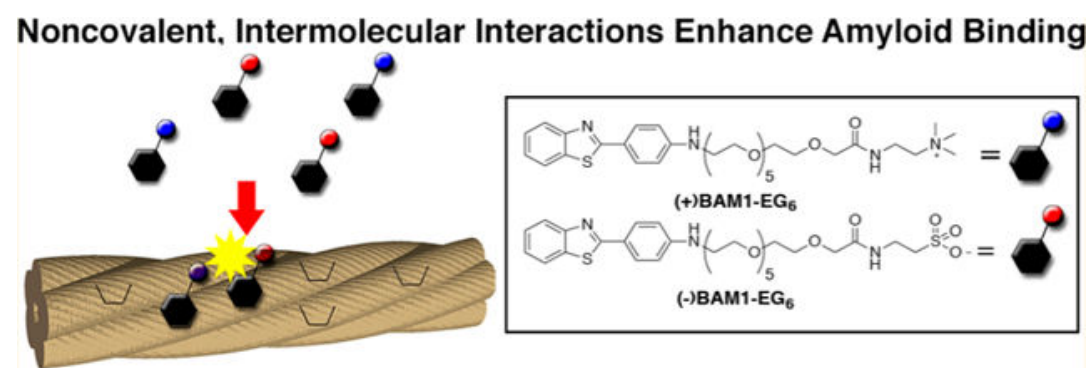

\section{Keywords}

Amyloid- $\beta$; cooperativity; benzothiazole; aggregation; $\alpha$-synuclein

The conversion of soluble proteins into amyloids, proteinaceous aggregates with high cross$\beta$ sheet structure, is associated with a broad range of neuro-degenerative disorders ${ }^{1,2}$ such as Alzheimer's disease $^{3}(\mathrm{AD})$ or Parkinson's disease ${ }^{4}(\mathrm{PD})$, as well as non-neuropathic diseases such as type II diabetes ${ }^{5}$ and sexually transmitted diseases such as human immunodeficiency virus (HIV). ${ }^{6}$ As the list of amyloid-associated diseases continues to grow, ${ }^{7}$ methods to target these natural materials with small molecules for disease prevention, treatment, and/or diagnosis continues to be of increasing fundamental importance.

We previously reported the design and synthesis of several oligo(ethylene glycol) derivatives of benzothiazole aniline (BTA) that are capable of targeting amyloid aggregates with mid to high nanomolar affinity. ${ }^{8-10}$ In addition to demonstrating the capability of these molecules to protect neuroblastoma cells from the toxicity of Alzheimer's-related amyloid- $\beta(\mathrm{A} \beta)$ aggregates, ${ }^{10,11}$ we showed that these molecules were also capable of targeting the naturally abundant amyloid Semen-derived Enhancer of Virus Infection (SEVI) and neutralizing its capability to enhance HIV transmission in cervical cells. While these studies reveal some exciting potential opportunities for amyloid targeting agents in disease treatment and prevention, novel approaches to further improve amyloid binding affinities are still needed to improve potency.

In order to develop a method to improve the binding of synthetic molecules to amyloid targets, we $\mathrm{e}^{12,13}$ and others ${ }^{14}$ previously reported the development of oligomeric ${ }^{12}$ and polymeric ${ }^{13}$ BTA derivatives that were designed to simultaneously bind to multiple binding sites (i.e., multivalent binding) along the surface of amyloid fibrils (Figure 1a); adjacent binding sites on amyloid fibrils for BTA moieties have been estimated to be as close as $2 \mathrm{~nm}$ apart. ${ }^{15}$ As expected, the oligomeric BTA derivatives exhibited a trend of increasing binding to aggregated $\mathrm{A} \beta$ peptides as a function of increasing valence number. ${ }^{12}$ While this multivalent approach to the design of amyloid binding molecules led to significant improvements in overall binding compared to monomeric BTA molecules, some significant drawbacks ${ }^{16,17}$ to this previous approach are (1) the difficulty of synthesis, ${ }^{12}$ (2) the poor aqueous solubility of the oligomers, ${ }^{18}$ and (3) the large size of the oligomeric compounds that introduce potential challenges for biocompatibility. ${ }^{19}$ In order to address these challenges while also building upon the promising use of cooperative interactions for improving amyloid binding of synthetic molecules, here we designed and synthesized two 
charged derivatives of the known amyloid-binding benzothiazole molecule, BAM1-EG 6 (Figure 1b), ${ }^{10,20}$ which we hypothesized could introduce cooperative noncovalent interactions between molecules bound to adjacent binding sites along the surface of amyloids, thereby improving amyloid binding while eliminating the need for covalent linkages between amyloid-binding moieties.

In this proof-of-concept study, we used electrostatic interactions between small molecules as a simple demonstration of an approach to introduce cooperativity in the binding to amyloid targets (Figure 1b). We incorporated choline and sulfonate groups into the parent BAM1$\mathrm{EG}_{6}$ compounds since they have been widely used to install positive and negative charges, respectively, on molecules due to their capability to retain essentially permanent charges across a broad range of $\mathrm{pH}^{21}$ The negatively charged (-)BAM1-EG 6 and positively charged (+)BAM1-EG 6 (Figure 1b) were prepared from BAM1-EG ${ }_{6}^{10,20}$ through standard $\mathrm{S}_{\mathrm{N}} 2$ and amide coupling procedures (see Scheme $\mathrm{S} 1$ and the Supporting Information for details on the synthesis and characterization of these charged compounds). We used previously reported $^{12}$ BTA monomer (1) and dimer (2) (Figure 1a) as controls in this study to compare multivalent versus noncovalent interactions as strategies for designing high affinity binding agents to amyloid targets.

With the charged BAM1-EG 6 derivatives in hand, we compared the binding of pure BAM1$\mathrm{EG}_{6}$ to the binding of a 1:1 mixture of (+)BAM1-EG 6 :(-)BAM1-EG 6 [referred to as $(+/-)$ BAM1-EG $_{6}$ for short] to aggregated $\mathrm{A} \beta$ peptides utilizing a previously reported centrifugation binding assay. ${ }^{12,22}$ This intrinsic fluorescence assay was performed under equilibrium binding conditions (Figure S1). Here, we found a significantly stronger binding interaction between aggregated $\mathrm{A} \beta$ and the $(+/-) \mathrm{BAM}_{1}-\mathrm{EG}_{6}$ mixture $\left(K_{\mathrm{d}}=20 \mathrm{nM}\right)$ compared to the binding of the uncharged BAM1-EG $6\left(K_{\mathrm{d}}=170 \mathrm{nM}\right)$ (Table 1, Figure S2). In order to compare the strategies for improving the binding of small molecules to amyloid targets using multivalent interactions versus non-covalent, intermolecular interactions (Figure 1), we calculated the enhancement factor, fi, proposed by Whitesides and co-workers $\left[\beta=K_{\mathrm{a}} \text { (multi) } / K_{\mathrm{a}} \text { (mono) }\right]^{16,17}$ for both cases, which provides an estimate of the benefit of multimeric binding interactions in systems with unknown number of total binding sites $(N)$ (as is the case with a heterogeneous mixture of amyloid aggregates). Here, we calculated an enhancement factor, $\beta$, of 10 for binding of the (+/-)BAM1-EG 6 mixture to aggregated $\mathrm{A} \beta$ relative to $\mathrm{BAM} 1-\mathrm{EG}_{6}$ (Table 1), which was slightly better than the enhancement factor calculated for the binding of the covalently linked BTA dimer $2\left(K_{\mathrm{d}}=29 \mathrm{nM}\right)$ versus BTA monomer $1\left(K_{\mathrm{d}}=235 \mathrm{nM}\right)$ to aggregated $\mathrm{A} \beta(\beta=8$, Figure $\mathrm{S} 3)$.

Next, we examined the degree of cooperativity for the binding of BTA dimer 2 and for the binding of the (+/-)BAM1-EG 6 mixture to $\mathrm{A} \beta$ aggregates by estimating the Hill coefficients from the saturation binding curves of $A \beta$ aggregates bound by the compounds (Figure 2, also see Figures S2 and S3). ${ }^{23}$ The analysis revealed that the binding of dimer 2 to A $\beta$ aggregates was slightly negatively cooperative, with a Hill coefficient of $h=0.8$ ( $p=0.005$, compared to $h=1)$. This result is consistent with the observed negative cooperativity typically found in most reported natural and unnatural multivalent binding systems due to various factors such as unfavorable geometric strain or entropic cost due to the structural constraint of linkers used to covalently join ligands and receptors. ${ }^{16}$ In contrast to the results 
with BTA dimer 2, we found that the (+/-)BAM1-EG 6 mixture exhibited positive cooperativity upon binding to $A \beta$ aggregates, with a Hill coefficient of $h=3.3$; this result was significantly different from the observed Hill coefficient for both the dimer $\mathbf{2}(p=$ $0.001)$ and neutral parent compound, $\mathrm{BAM}^{-\mathrm{EG}_{6}}(p=0.02)$, as determined by unpaired $t$ test (Table 1). In order to provide a qualitative visual representation for these differences in cooperativity, Figure 2 shows an overlay of the normalized binding curves of (+/-)BAM1$\mathrm{EG}_{6}$ and dimer 2 to aggregated $\mathrm{A} \beta$ peptides, where the increasing steepness of the sigmoidal curve reflects the larger Hill coefficient for the binding of the mixture of charged BTA molecules compared to dimer 2.

As a control, we also assessed the binding of pure (+) or (-) BAM1-EG 6 to $\mathrm{A} \beta$ aggregates. Since the $\mathrm{pI}$ of $\mathrm{A} \beta$ has been reported to be $\sim 5.5,{ }^{24}$ we performed all binding studies at $\mathrm{pH}$ 5.5 to minimize electrostatic interactions between the charged BAM agents and aggregated A $\beta$ (Figure S4). Surprisingly, under these conditions we found that pure (+)BAM1-EG 6 exhibited a $K_{\mathrm{d}}=290 \mathrm{nM}$ to A $\beta$ aggregates, whereas pure (-)BAM1-EG 6 exhibited a $K_{\mathrm{d}}=40$ $\mathrm{nM}$ to $\mathrm{A} \beta$ aggregates (Figure $\mathrm{S} 4$ ) (compared to a $K_{\mathrm{d}}=170 \mathrm{nM}$ for uncharged BAM1-EG 6 ), suggesting that some electrostatic interactions between the charged small molecules and the (apparently positively charged) amyloid surface could not be completely avoided at $\mathrm{pH}$ 5.5. However, the low enhancement factor $(\beta=0.6$ and $\beta=4)$, the lack of significant positive

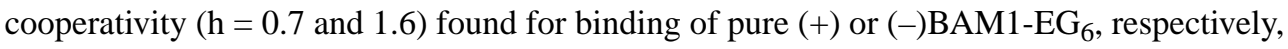
as well as the tighter apparent binding of the (+/-)BAM1-EG 6 mixture $(20 \mathrm{nM})$ to $\mathrm{A} \beta$ aggregates compared to ether pure charged BAM derivative ( $40 \mathrm{nM}$ or $290 \mathrm{nM}$ ) suggests that the intermolecular noncovalent interactions between oppositely charged BAM agents in the 1:1 mixture of (+/-)BAM1-EG 6 play an important role in the observed increased apparent binding of this mixture to the amyloid surface.

In order to provide additional experimental evidence that electrostatic interactions play a role in the binding of (+/-)BAM1-EG 6 to $\mathrm{A} \beta$ aggregates, we examined the binding of (+/-)BAM1-EG 6 in $\mathrm{pH} 5.5$ water containing high salt concentrations (i.e., $500 \mathrm{mM} \mathrm{NaCl}$ ), which we expect would diminish electrostatic interactions. ${ }^{25}$ As anticipated, under high salt conditions we observed a significantly lower apparent binding affinity for (+/-)BAM1-EG 6 to $\mathrm{A} \beta$ aggregates compared to when no $\mathrm{NaCl}$ was added (Table 1). Additionally, the positive cooperativity that was observed for the (+/-)BAM1-EG 6 mixture in pure water was eliminated in the presence of high salt, exhibiting a Hill coefficient that was similar (i.e., not significant by unpaired $t$ test) to neutrally charged BAM1-EG 6 bound to aggregated $\mathrm{A} \beta$ (Table 1). Taken together, the observed decrease in apparent affinity and cooperativity for binding of (+/-)BAM1-EG 6 to $\mathrm{A} \beta$ aggregates under high salt conditions suggests that electrostatic interactions play a dominant role for the improved binding of (+/-)BAM1-EG 6 to $\mathrm{A} \beta$ compared to $\mathrm{BAM} 1-\mathrm{EG}_{6}$ in pure water.

To examine whether the use of noncovalent, intermolecular interactions could improve the binding of BTA derivatives to amyloids derived from peptides or proteins other than $\mathrm{A} \beta$, we next tested the binding of (+/-)BAM1-EG 6 to a preparation of aggregated $a$-synuclein ( $a \mathrm{~S}$ ) at $\mathrm{pH} 5$ (the $\mathrm{pI}$ of $a \mathrm{~S}$ has been reported to be $\sim 4.7^{26}$ ), a protein associated with Lewy bodies found in patients with Parkinson's disease. As was the case with $\mathrm{A} \beta$ aggregates, we found that $(+/-)$ BAM1-EG 6 bound stronger $\left(K_{\mathrm{d}}=400 \mathrm{nM}\right)$ to aggregated $a \mathrm{~S}$ compared to the 
uncharged parent compound, BAM1-EG $6\left(K_{\mathrm{d}}=1100 \mathrm{nM}\right)$, with an estimated enhancement factor $\beta$ of 3 (Table 1, Figure S5). Under high salt conditions, this enhancement of apparent binding of (+/-)BAM1-EG 6 to aggregated $a \mathrm{~S}$ was abolished and the apparent binding was comparable of BAM1-EG 6 . Furthermore, a Hill coefficient of $h=3.8$ (with no added salt) suggested that (+/-)BAM1-EG 6 exhibited positive cooperativity for binding to aggregated

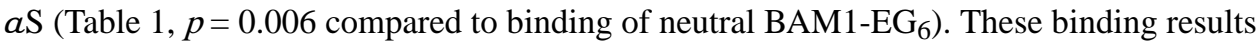
with $a \mathrm{~S}$ further support that noncovalent, electrostatic interactions between oppositely charged BTA derivatives can improve the apparent binding of small molecules to multiple (here, two) amyloidogenic protein aggregates.

In conclusion, we have demonstrated that noncovalent interactions between synthetic molecules can be used to improve their binding to amyloid targets. We show that a 1:1 mixture of negatively charged (-)BAM1-EG 6 and positively charged (+)BAM1-EG 6 exhibited a 10 -fold enhancement of binding to aggregated $\mathrm{A} \beta$ peptides and a 3 -fold enhancement for binding to aggregated $a \mathrm{~S}$ proteins compared to the neutrally charged parent compound, BAM1-EG 6 . The observed enhancement in binding was substantially reduced in the presence of high salt, supporting the significant role of electrostatic interactions in the binding of (+/-)BAM1-EG 6 to the amyloid surface. We hypothesize that differences in average binding site densities for small molecules on different amyloid compositions ${ }^{15,27}$ could play a role in influencing the extent of improved binding between molecules bound to nearby sites on an amyloid surface (Figure S6). While it remains to be seen, such differences in average binding site densities may potentially be further exploited to improve the targeting of one amyloid composition over another using a similar approach as described here. Furthermore, we show that using noncovalent interactions between monomeric amyloid-binding molecules led to the same degree of enhancement in binding to aggregated $\mathrm{A} \beta$ as a covalently attached dimeric molecule $\mathbf{2}$, and demonstrated the possibility of using noncovalent interactions to impart positive cooperativity into the amyloid-binding properties of small molecules. Given that the affinity of BTA analogues for binding to amyloids was directly correlated with potency for reducing seminal amyloid-mediated HIV infection, ${ }^{12}$ the work presented here could lead to improved design of microbicide supplements for reducing transmission of certain sexually transmitted diseases. Current efforts are focused on exploring other noncovalent or reversible interactions (such as metal chelation) in the design of high affinity amyloid-binding small molecules to improve biocompatibility and targeting of amyloids associated with Alzheimer's and other amyloidrelated diseases.

\section{METHODS}

\section{Measurement ofthe Binding Affinity to Aggregated $A \beta(1-42)$ or $a$-Synuclein $(a S)$.}

Binding of compounds to aggregated $\mathrm{A} \beta(1-42)$ and $a \mathrm{~S}$ was measured according to a previously described assay. ${ }^{8}$ Briefly, $200 \mu \mathrm{L}$ of various concentrations of BTA/BAM compounds in DI $\mathrm{H}_{2} \mathrm{O}$ or $500 \mathrm{mM} \mathrm{NaCl} / \mathrm{DI} \mathrm{H} 2 \mathrm{O}$ (high salt conditions) were incubated in the absence or presence of $10 \mu \mathrm{g}$ of preaggregated amyloid (total volume $220 \mu \mathrm{L}$ ). Solutions were allowed to equilibrate overnight at room temperature. Samples were then centrifuged at $16000 \mathrm{~g}$ for $20 \mathrm{~min}$ at $4{ }^{\circ} \mathrm{C}$. The supernatants were removed and the pellet was resuspended 
in $220 \mu \mathrm{L}$ of fresh DI H2O or $500 \mathrm{mM} \mathrm{NaCl}$. Fluorescence of the bound molecule was determined using a spectrofluorometer (Photon Technology International, Inc., Birmingham, $\mathrm{NJ})$. Each experiment was repeated at least three times, and error bars denote standard deviation from the mean. Graphs shown in Figures S1-S5 were fit using the following onesite specific binding algorithm with Hill slope to determine $K_{d}: Y=B_{\max } X^{h} /\left(K_{d}{ }^{h}+X^{h}\right)$, where $X$ is the concentration of small molecule, $Y$ is the specific binding intensity, $B_{\max }$ is the apparent maximal observable fluorescence upon binding to $\mathrm{A} \beta / a \mathrm{~S}$, and $h$ is the Hill slope. Data was processed using Origin 7.0 (MicroCal Software, Inc., Northampton, MA) and GraphPad Prism 6. For statistics comparing Hill coefficients, the unpaired $t$ test with Welch's correction was used and calculated from GraphPad Prism 6 (GraphPad Software, Inc. La Jolla, CA).

\section{Supplementary Material}

Refer to Web version on PubMed Central for supplementary material.

\section{ACKNOWLEDGMENTS}

We thank Dr. Eric Luth for providing the plasmid for $a$-synuclein as a gift.

Funding

Research reported in this publication was supported by the National Institute On Aging of the National Institutes of Health under Award Number R01AG053577.

\section{REFERENCES}

(1). Eisenberg D, and Jucker M (2012) The amyloid state of proteins in human diseases. Cell 148, 1188-203. [PubMed: 22424229]

(2). Knowles TPJ, Vendruscolo M, and Dobson CM (2014) The amyloid state and its association with protein misfolding diseases. Nat. Rev. Mol Cell Biol. 15, 384-96. [PubMed: 24854788]

(3). Hardy J, and Selkoe DJ (2002) The amyloid hypothesis of Alzheimer's disease: progress and problems on the road to therapeutics. Science 297, 353-6. [PubMed: 12130773]

(4). Moore DJ, West AB, Dawson VL, and Dawson TM (2005) Molecular Pathophysiology of Parkinson's Disease. Annu. Rev. Neurosci 28, 57-87. [PubMed: 16022590]

(5). Hull RL, Westermark GT, Westermark P, and Kahn SE (2004) Islet amyloid: A critical entity in the pathogenesis of type 2 diabetes. J. Clin. Endocrinol. Metab. 89, 3629-3643. [PubMed: 15292279]

(6). Münch J, Rucker E, Standker L, Adermann K, Goffinet C, Schindler M, Wildum S, Chinnadurai R, Rajan D, Specht A, Giménez-Gallego G, Sanchez PC, Fowler DM, Koulov A, Kelly JW, Mothes W, Grivel JC, Margolis L, Keppler OT, Forssmann WG, and Kirchhoff F (2007) SemenDerived Amyloid Fibrils Drastically Enhance HIV Infection. Cell 131, 1059-1071. [PubMed: 18083097]

(7). Chiti F, and Dobson CM (2006) Protein misfolding, functional amyloid, and human disease. Annu. Rev. Biochem. 75, 333-66. [PubMed: 16756495]

(8). Olsen JS, Brown C, Capule CC, Rubinshtein M, Doran TM, Srivastava RK, Feng C, Nilsson BL, Yang J, and Dewhurst S (2010) Amyloid-binding small molecules efficiently block SEVI (semen-derived enhancer of virus infection)-and semen-mediated enhancement of HIV-1 infection. J. Biol. Chem. 285, 35488-96. [PubMed: 20833717]

(9). Inbar P, Li CQ, Takayama SA, Bautista MR, and Yang J (2006) Oligo(ethylene glycol) derivatives of thioflavin $\mathrm{T}$ as inhibitors of protein-amyloid interactions. ChemBioChem 7, 1563-6. [PubMed: 16927253] 
(10). Cifelli JL, Chung TS, Liu H, Prangkio P, Mayer M, and Yang J (2016) Benzothiazole Amphiphiles Ameliorate Amyloid $\beta$-Related Cell Toxicity and Oxidative Stress. ACS Chem. Neurosci. 7, 682-8. [PubMed: 27055069]

(11). Habib LK, Lee MTC, and Yang J (2010) Inhibitors of catalase-amyloid interactions protect cells from beta-amyloid-induced oxidative stress and toxicity. J. Biol. Chem. 285, 38933-43. [PubMed: 20923778]

(12). Capule CC, Brown C, Olsen JS, Dewhurst S, and Yang J (2012) Oligovalent amyloid-binding agents reduce SEVI-mediated enhancement of HIV-1 infection. J. Am. Chem. Soc. 134, 905-8. [PubMed: 22239120]

(13). Sheik DA, Brooks L, Frantzen K, Dewhurst S, and Yang J (2015) Inhibition of the enhancement of infection of human immunodeficiency virus by semen-derived enhancer of virus infection using amyloid-targeting polymeric nanoparticles. ACS Nano 9, 1829-36. [PubMed: 25619867]

(14). Qin L, Vastl J, and Gao J (2010) Highly sensitive amyloid detection enabled by thioflavin T dimers. Mol. BioSyst. 6, 1791-5. [PubMed: 20614050]

(15). Lockhart A, Ye L, Judd DB, Merritt AT, Lowe PN, Morgenstern JL, Hong G, Gee AD, and Brown J (2005) Evidence for the presence of three distinct binding sites for the thioflavin T class of Alzheimer's disease PET imaging agents on beta-amyloid peptide fibrils. J. Biol. Chem. 280, 7677-84. [PubMed: 15615711]

(16). Krishnamurthy VM, Estroff LA, and Whitesides GM (2006) Multivalency in Ligand Design In Fragment-based Approaches in Drug Discovery (Jahnke W, and Erlanson D, Eds.), Wiley-VCH Verlag $\mathrm{GmbH} \& \mathrm{Co}$, Weinheim.

(17). Mammen M, Choi S-K, and Whitesides GM (1998) Polyvalent Interactions in Biological Systems: Implications for Design and Use of Multivalent Ligands and Inhibitors. Angew. Chem., Int. Ed. 37 2754-2794.

(18). Savjani KT, Gajjar AK, and Savjani JK (2012) Drug solubility: importance and enhancement techniques. ISRN Pharm. 2012, 195727. [PubMed: 22830056]

(19). Pajouhesh H, and Lenz GR (2005) Medicinal chemical properties of successful central nervous system drugs. NeuroRx 2, 541-53. [PubMed: 16489364]

(20). Cifelli JL, Dozier L, Chung TS, Patrick GN, and Yang J (2016) Benzothiazole Amphiphiles Promote the Formation of Dendritic Spines in Primary Hippocampal Neurons. J. Biol. Chem. 291, 11981-11992. [PubMed: 27022020]

(21). Liu R, Ed. (2008) Water-Insoluble Drug Formulation, 2nd ed, CRC Press, Boca Raton.

(22). LeVine H III (2005) Multiple ligand binding sites on Abeta(1-40) fibrils. Amyloid 12, 5-14. [PubMed: 16076606]

(23). Weiss J (1997) The Hill equation revisited : uses and misuses. FASEB J. 11, 835-841. [PubMed: 9285481]

(24). Rauk A (2009) The chemistry of Alzheimer's disease. Chem. Soc. Rev. 38, 2698. [PubMed: 19690748]

(25). Capone R, Blake S, Restrepo MR, Yang J, and Mayer M (2007) Designing Nanosensors Based on Charged Derivatives of Gramicidin A. J. Am. Chem. Soc. 129, 9737-9745. [PubMed: 17625848]

(26). Vigneswara V, Cass S, Wayne D, Bolt EL, Ray DE, and Carter WG (2013) Molecular Ageing of Alpha-and Beta-Synucleins: Protein Damage and Repair Mechanisms. PLoS One 8, e61442. [PubMed: 23630590]

(27). Ye L, Velasco A, Fraser G, Beach TG, Sue L, Osredkar T, Libri V, Spillantini MG, Goedert M, and Lockhart A (2008) In vitro high affinity alpha-synuclein binding sites for the amyloid imaging agent PIB are not matched by binding to Lewy bodies in postmortem human brain. J. Neurochem. 105, 1428-37. [PubMed: 18221373] 


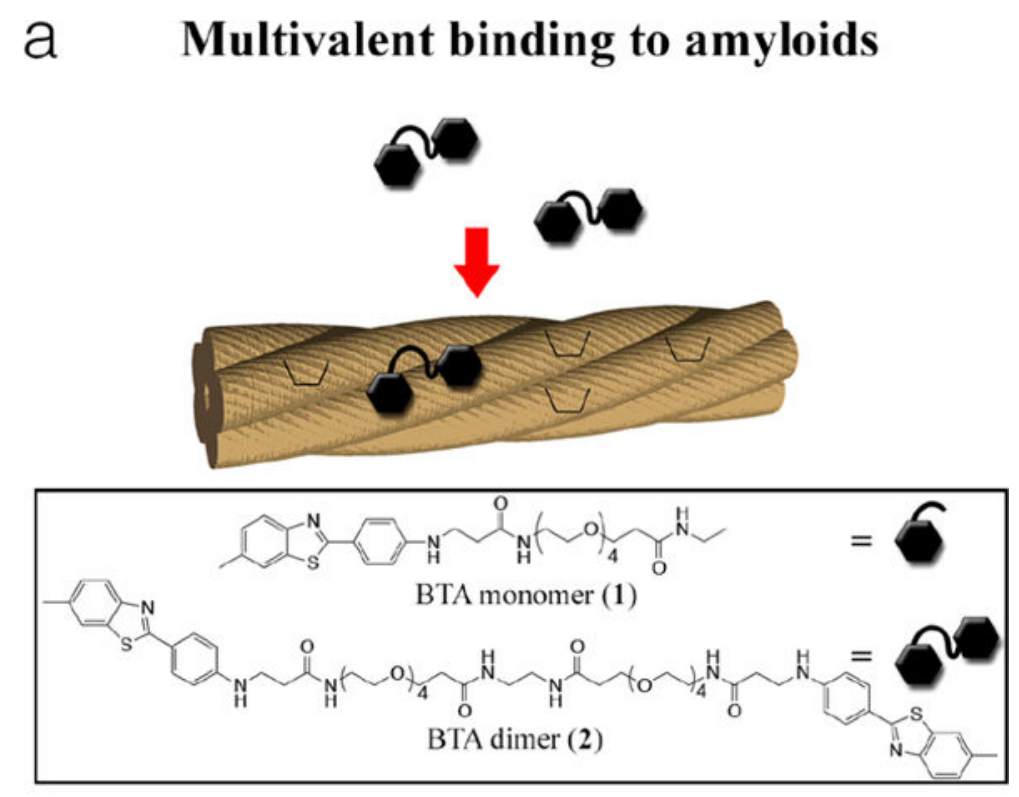

\section{b Enhanced amyloid binding due to noncovalent, intermolecular interactions}

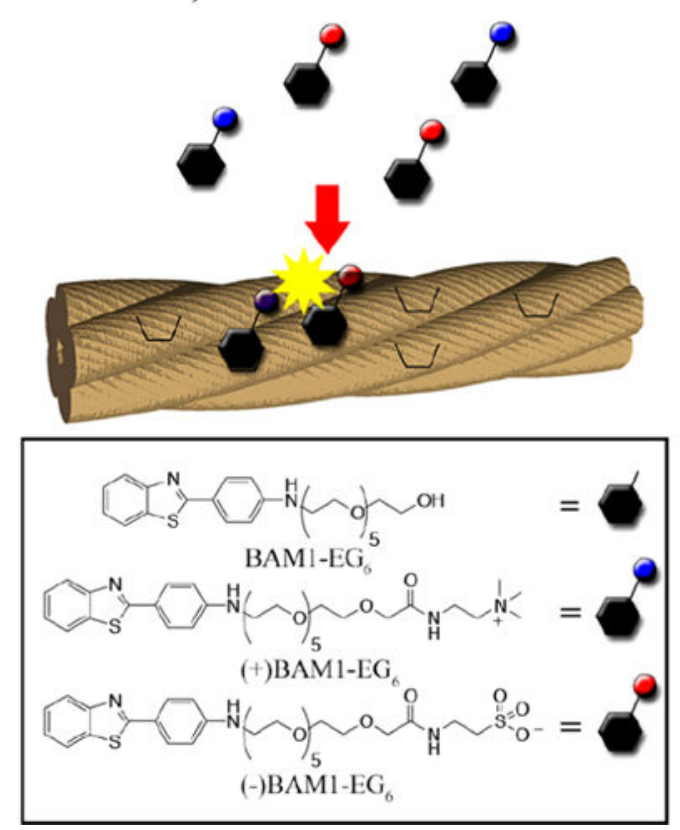

Figure 1.

Comparison of strategies that use either multivalency (a) or noncovalent electrostatics (b) to improve the binding of various BTA derivatives to amyloid fibrils. 


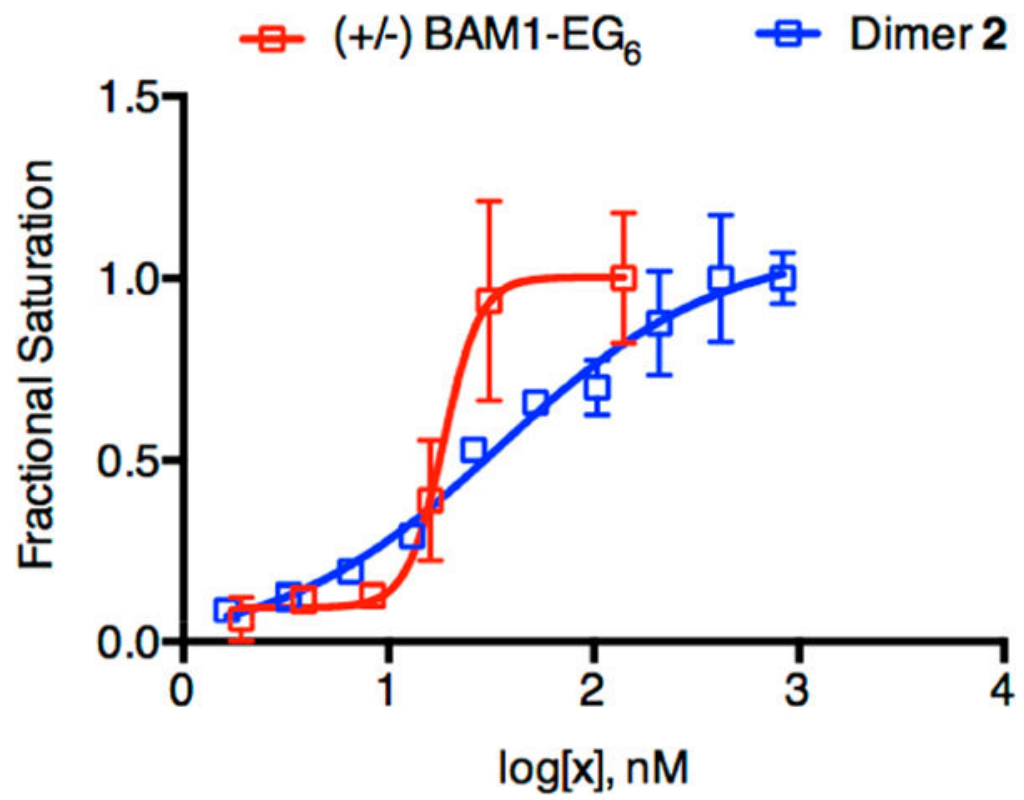

Figure 2.

Comparison of the normalized binding saturation plots of the covalently linked BTA dimer 2 and noncovalent mixture of (+/-)BAM1-EG 6 to aggregated $\beta$-amyloid (1-42) peptides.

Fluorescence intensities were normalized for each respective binding curve in order to estimate fractional saturation of binding sites. 


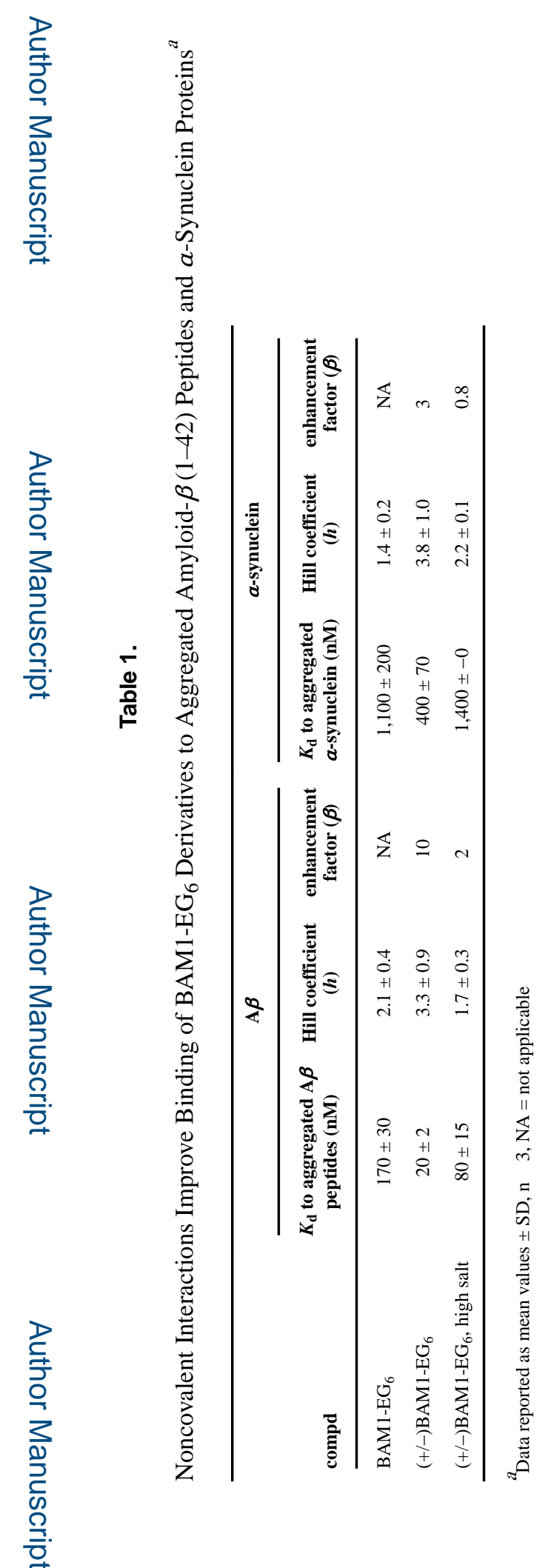

ACS Chem Neurosci. Author manuscript; available in PMC 2019 May 30. 